\title{
Properties of the Geometric and Related Processes
}

\author{
W. John Braun, ${ }^{1}$ Wei Li, ${ }^{2}$ Yiqiang Q. Zhao ${ }^{3}$ \\ ${ }^{1}$ Department of Statistical and Actuarial Sciences, University of Western Ontario, London, Canada N6A 5B7 \\ ${ }^{2}$ Department of Electrical and Computer Engineering, The University of Toledo, Mail Stop 308, \\ Toledo, Ohio 43606-3390 \\ ${ }^{3}$ School of Mathematics and Statistics, Carleton University, Ottawa, Canada K1S 5B6
}

Received 4 April 2001; revised 1 April 2005; accepted 28 April 2005

DOI 10.1002/nav.20099

Published online 14 July 2005 in Wiley InterScience (www.interscience.wiley.com).

\begin{abstract}
Some properties of the geometric process are studied along with those of a related process which we propose to call the $\alpha$-series process. It is shown that the expected number of counts at an arbitrary time does not exist for the decreasing geometric process. The decreasing version of the $\alpha$-series process does have a finite expected number of counts, under certain conditions. This process also has the same advantages of tractability as the geometric process; it exhibits some properties which may make it a useful complement to the increasing geometric process. In addition, it may be fit to observed data as easily as the geometric process. Applications in reliability and stochastic scheduling are considered in order to demonstrate the versatility of the alternative model. () 2005 Wiley Periodicals, Inc. Naval Research Logistics 52: 607-616, 2005.
\end{abstract}

Keywords: geometric process; reliability; renewal theory; birth processes; stochastic scheduling

\section{INTRODUCTION}

Consider a machine which operates for a random amount of time before being repaired and then operates again until the next repair is required, continuing to alternate between periods of operation and repair. Under many circumstances, the times between repairs (uptimes) would seem to most appropriately be modeled as a stochastically decreasing sequence of random variables (e.g., Ross [9]), since the machine may not always be repaired as good as new. For similar reasons, the sequence of periods during which the machine is being repaired (downtimes) may best be modeled as a stochastically increasing sequence of random variables.

In this paper, we consider two simple stochastic processes for modeling such deteriorating repairable machines. Both processes are generalizations of the renewal process. The first is the geometric process, proposed by Lam [6]. The second is a related process which we propose as a counterpart to the geometric process.

Correspondence to: W.J. Braun (braun@stats.uwo.ca)
Lam [6] has investigated many important theoretical properties of the geometric process. The process gives tractable solutions for some reliability problems (Lam [5] and Lam and Zheng [8]). Given a set of data, statistical inference can be easily carried out for this process (Lam [7]). Thus, the model is an attractive candidate for reliability problems involving nonstationary processes. Furthermore, no specific distributional assumptions other than some moment conditions are required for the uptimes or downtimes, so the model should have wide applicability.

The model proposed in this paper shares many of the advantages of the geometric process, including simplicity. In the succeeding sections of this paper, we shall examine some of its theoretical properties, as well as its applicability to reliability and scheduling problems. We shall also show that statistical inference for this new process is as straightforward as for the geometric process.

The outline of the paper is as follows. In the next section, we describe the two models. Section 3 is concerned with the existence and nonexistence of the first moment of each of the process counts. Section 4 is devoted to the special case of exponential uptimes or downtimes, and is meant to convey information about the qualitative behavior of the expected counts $(N(t))$ in each case. Section 5 deals with the 
problem of statistical inference-estimation of the process parameters from observed data. Section 6 describes how the expected values of $N(t)$ can be numerically approximated for each process. Section 7 describes two reliability and scheduling applications. Section 8 summarizes our findings.

\section{THE TWO MODELS}

Suppose $Y_{1}, Y_{2}, \cdots$ is a sequence of independent and identically distributed random variables with distribution function $F(y)$ and mean $m<\infty$.

The geometric process can be constructed as follows. Let

$$
X_{i}^{G}=\frac{Y_{i}}{a^{i-1}}, \quad i=1,2,3, \cdots
$$

for some $a>0$. If

$$
S_{0}^{G}=0 \quad \text { and } \quad S_{n}^{G}=\sum_{i=1}^{n} X_{i}^{G}
$$

then

$$
N^{G}(t)=\sup \left\{n: S_{n}^{G} \leq t\right\} \quad(t \geq 0)
$$

is defined as a geometric process with parameter $a$.

When $a>1$, Lam [5] observed that the $X_{i}^{G}$, s form a stochastically decreasing sequence, and thus proposed $N^{G}(t)$ with $a>1$ as a possible model for the number of machine uptimes by time $t$. When $a<1$, the $X_{i}^{G}$, s are stochastically increasing. In this case, Lam proposed $N^{G}(t)$ as a model for downtimes. When $a=1, N^{G}(t)$ is a renewal process.

A related process can be constructed as follows. Let

$$
X_{i}^{A}=\frac{Y_{i}}{i^{\alpha}}, \quad i=1,2,3, \ldots,
$$

where $\alpha$ is a real-valued parameter. Set

$$
S_{0}^{A}=0 \quad \text { and } \quad S_{n}^{A}=\sum_{i=1}^{n} X_{i}^{A},
$$

and define the point process

$$
N^{A}(t)=\sup \left\{n: S_{n}^{A} \leq t\right\} .
$$

We shall call the process $N^{A}(t)$ an $\alpha$-series process. When $\alpha=0, N^{A}(t)$ is a renewal process. If $\alpha>0,\left\{X_{i}^{A}\right.$, $i \geq 1\}$ is a stochastically decreasing sequence which can be used as a model for machine uptimes. If $\alpha<0,\left\{X_{i}^{A}, i \geq\right.$ $1\}$ is stochastically increasing. In this case, this process can be used as a model for machine downtimes.

It should also be noted that when the $Y_{i}$ 's are exponentially distributed and $\alpha=1, N^{A}(t)$ is a linear birth process and, when $\alpha=0, N^{A}(t)$ is a Poisson process. For other values of $\alpha, N^{A}(t)$ is a birth process.

\section{THE EXISTENCE OF $E\left[N^{G}(t)\right]$ AND $E\left[N^{A}(t)\right]$}

The usefulness of the geometric process and its relatives in reliability and scheduling applications depends upon the first moment being finite. Many of the equations which result from such optimization problems contain terms involving this moment explicitly. Furthermore, numerical methods are usually required to calculate approximations for this moment; clearly, one should not attempt such a calculation if the moment does not exist. Thus, it is important to establish easy-to-verify conditions for the existence or nonexistence of $E\left[N^{G}(t)\right]$ and $E\left[N^{A}(t)\right]$.

Lam [6] considered this problem for the geometric process and derived the following analogue of the renewal equation,

$$
E\left[N^{G}(t)\right]=F(t)+\int_{0}^{t} E\left[N^{G}(a(t-x))\right] d F(x),
$$

and gave conditions under which a unique solution $E\left[N^{G}(t)\right]$ to this equation exists. Verifying these conditions amounts to checking whether a sequence of approximate solutions is uniformly bounded in any finite interval. Lam concluded that $E\left[N^{G}(t)\right]$ is finite when $a \leq 1$, and he gave an example in which these conditions are not satisfied and the moment is not finite in the case where $a>1$.

An additional necessary condition for the finiteness of $E[N(t)]$ when $a \leq 1$ is $F(0)<1$. Furthermore, Lam's example actually typifies the behavior of $E\left[N^{G}(t)\right]$ when $a>1$ as we now show.

THEOREM 1: If $a>1$ and $F(\varepsilon)>0$ for all $\varepsilon>0$, then $E\left[N^{G}(t)\right]$ is infinite for all $t>0$.

\section{PROOF:}

$$
E\left[X_{i}^{G}\right]=\frac{E\left[Y_{i}\right]}{a^{i-1}}=\frac{m}{a^{i-1}} .
$$

Therefore, 


$$
E\left[\sum_{i=1}^{\infty} X_{i}^{G}\right]=\frac{m a}{a-1}<\infty,
$$

and there exists $\varepsilon>0$ such that, for any $n$,

$$
P\left(\sum_{i=1}^{n} X_{i}^{G} \leq \frac{a m}{a-1}\right) \geq P\left(\sum_{i=1}^{\infty} X_{i}^{G} \leq \frac{a m}{a-1}\right)>\varepsilon .
$$

Since

$$
N^{G}(t) \geq n \quad \text { iff } \quad \sum_{i=1}^{n} X_{i}^{G} \leq t
$$

we have

$$
P\left(N^{G}\left(\frac{a m}{a-1}\right) \geq n\right)>\varepsilon
$$

for all $n$. Hence,

$$
E\left[N^{G}\left(\frac{a m}{a-1}\right)\right]=\infty
$$

and

$$
E\left[N^{G}(t)\right]=\infty
$$

for all $t \geq t_{1}=a m /(a-1)$. Set $t_{2}=t_{1} / a+\eta$, where $0<$ $\eta \leq t_{1} / a$. Then,

$$
\begin{aligned}
E\left[N^{G}\left(t_{2}\right)\right] & =\int_{0}^{t_{2}} E\left[N^{G}\left(a\left(t_{2}-x\right)\right)\right] d F(x)+F\left(t_{2}\right) \\
\geq & \int_{0}^{t_{1} / a} E\left[N^{G}\left(t_{1}+a \eta-a x\right)\right] d F(x)+F\left(t_{2}\right) \\
& \geq \int_{0}^{\eta} E\left[N^{G}\left(t_{1}+a \eta-a x\right)\right] d F(x)+F\left(t_{2}\right)=\infty
\end{aligned}
$$

since

$$
t_{1}-a x+a \eta>t_{1}
$$

when $0<x<\eta$. Therefore,

$$
E\left[N^{G}(t)\right]=\infty
$$

for all $t>t_{1} / a$.

Iterating this argument $n$ times, we can see that

$$
E\left[N^{G}(t)\right]=\infty
$$

for all $t>t_{1} / a^{n}$. Since $a>1$, we can make $t_{1} / a^{n}$ arbitrarily small.

REMARK 1: If $F(\varepsilon)=0$ for some $\varepsilon>0$, it is possible for $E\left[N^{G}(t)\right]$ to be finite for small $t$, but the above proof can be modified to show that $E\left[N^{G}(t)\right]$ is infinite for all $t>\tau$, where $\tau$ is such that $F(\tau)>0$.

REMARK 2: The condition $F(\varepsilon)>0$ holds for most frequently occurring distributions in reliability applications: for example, exponential, gamma, and Weibull.

For the $\alpha$-series process, $E\left[N^{A}(t)\right]<\infty$ in the increasing case, as well as in the decreasing case, provided that the random variable $Y$ has a sufficient number of moments.

THEOREM 2: Suppose $F(0)<1$.

1. If $\alpha<0$, then $E\left[N^{A}(t)\right]$ is finite for all $t>0$.

2. If $0 \leq \alpha \leq 1 / 2$ and $E\left[\left|Y_{i}\right|^{2+\delta}\right]<\infty$ for some $\delta>$ 0 , then $E\left[N^{A}(t)\right]$ is finite for all $t>0$.

3. If $1 / 2<\alpha<1$, and $E\left[Y_{i}^{(1+\delta) /(1-\alpha)}\right]<\infty$ for some $\delta>0$, then $E\left[N^{A}(t)\right]$ is finite for all $t>0$.

4. If $\alpha=1$, and all moments of $Y_{i}$ exist such that, for some fixed $\delta>0$,

$$
\prod_{i=1}^{n} E\left[e^{\left|Y_{i}-m\right|(1+\delta) / i}\right]
$$

is bounded, then $E\left[N^{A}(t)\right]$ is finite for all $t>0$.

5. If $\alpha>1$, and $F(\varepsilon)>0$ for some $\varepsilon>0$, then $E\left[N^{A}(t)\right]$ is infinite for all large enough $t$.

\section{PROOF:}

1. $\alpha<0$ : $S_{n}^{A}=\sum_{i=1}^{n} Y_{i} / i^{\alpha}>\sum_{i=1} Y_{i}$, so $N^{A}(t)<N(t)$, where $N(t)$ is an ordinary renewal process. Since $E[N(t)]<\infty$ (e.g., [4]), $E\left[N^{A}(t)\right]<\infty$.

2. $0 \leq \alpha \leq 1 / 2$ :

Take $p=2+\delta$, where $\delta>0$.

Using an inequality of Burkholder (e.g., (3.3.14) of Stout [11], p. 154), we have, for some constant $C$, 


$$
\begin{array}{r}
E\left[\left|S_{n}^{A}-\sum_{i=1}^{n} \frac{m}{i^{\alpha}}\right|^{p}\right] \leq C n^{p / 2-1} E\left[\sum_{i=1}^{n} \frac{\left|Y_{i}-m\right|^{p}}{i^{p \alpha}}\right] \\
=O\left(n^{-p / 2+1-\alpha p}\right) .
\end{array}
$$

For large enough $n, \sum_{i=1}^{n} m / i^{\alpha}>t$, since $F(0)<1$ ensures that $m$ is positive. For such $n$,

$$
\begin{aligned}
P\left(S_{n}^{A}<t\right) \leq P\left(\left|S_{n}^{A}-\sum_{i=1}^{n} \frac{m}{i^{\alpha}}\right|>\sum_{i=1}^{n} \frac{m}{i^{\alpha}}-t\right) \\
\leq \frac{E\left[\left|S_{n}^{A}-\sum_{i=1}^{n} \frac{m}{i^{\alpha}}\right|^{p}\right]}{\sum_{i=1}^{n}\left(\frac{m}{i^{\alpha}}-t\right)^{p}}=O\left(n^{-p / 2}\right)
\end{aligned}
$$

by Markov's inequality. Finally,

$$
E\left[N^{A}(t)\right]=\sum_{n=1}^{\infty} P\left(N^{A}(t) \geq n\right)=\sum_{n=1}^{\infty} P\left(S_{n}^{A}<t\right)<\infty .
$$

3. $1 / 2<\alpha<1$ :

For this case, we make use of the fact that $M_{n}=S_{n}^{A}$ $-\sum_{i=1}^{n} m / i^{\alpha}$ is an example of a martingale. Specifically, as for the case when $\alpha \leq 1 / 2$, we have

$$
\begin{array}{r}
E\left[N^{A}(t)\right]=\sum_{n=1}^{\infty} P\left(S_{n}^{A} \leq t\right) \leq P\left(\left|M_{n}\right| \geq m \sum_{i=1}^{\infty} \frac{1}{\bar{i}^{\alpha}}-t\right) \\
=\sum_{i=1}^{n} P\left(\left|M_{n}\right| \geq \frac{m n^{1-\alpha}}{1-\alpha}\right)+o\left(n^{1-\alpha}\right) \\
=\sum_{i=1}^{n} P\left(\left|M_{n}\right|^{(1+\delta) /(1-\alpha)} \geq n^{1+\delta}\left(\frac{m}{1-\alpha}\right)^{(1+\delta) /(1-\alpha)}\right) \\
+o\left(n^{1-\alpha}\right)
\end{array}
$$

for any fixed $\delta>0$. Markov's inequality can be applied to the last expression obtained, yielding the upper bound

$$
\begin{aligned}
& \sum_{i=1}^{n} E\left[\left|M_{n}\right|^{(1+\delta) /(1-\alpha)}\right] \frac{1}{n^{1+\delta}}\left(\frac{1-\alpha}{m}\right)^{(1+\delta) /(1-\alpha)} \\
&+o\left(n^{1-\alpha}\right) \leq \sum_{i=1}^{n} E\left[\sup _{1 \leq n<\infty}\left|M_{n}\right|^{(1+\delta) /(1-\alpha)}\right] \frac{1}{n^{1+\delta}} \\
& \times\left(\frac{1-\alpha}{m}\right)^{(1+\delta) /(1-\alpha)}+o\left(n^{1-\alpha}\right) .
\end{aligned}
$$

Now, Doob's inequality can be applied to yield the new upper bound

$C \sup _{1 \leq n<\infty} E\left[\left|M_{n}\right|^{(1+\delta) /(1-\alpha)}\right] \sum_{i=1}^{n} \frac{1}{n^{1+\delta}}\left(\frac{1-\alpha}{m}\right)^{(1+\delta) /(1-\alpha)}$

$$
+o\left(n^{1-\alpha}\right),
$$

where $C$ is a constant. This is finite (and hence, $E\left[N^{A}(t)\right]$ is also finite) if

$$
E\left[\left|M_{n}\right|^{(1+\delta) /(1-\alpha)}\right]<\infty .
$$

To demonstrate this, we invoke Rosenthal's inequality

$$
E\left[\left|S_{n}\right|^{p}\right] \leq C_{p}\left(\sum_{i=1}^{n} E\left[X_{i}^{2}\right]\right)^{p / 2}+C_{p} \sum_{i=1}^{n} E\left[\left|X_{i}\right|^{p}\right],
$$

which holds for some constant $C_{p}$, when $X_{1}$, $X_{2}, \ldots, X_{n}$ are independent random variables with $E\left[X_{i}\right]=0$, and $p \geq 1$. Applying this to our problem, we take $X_{i}=Y_{i} / i^{\alpha}-m / i^{\alpha}$, and $p=(1+$ $\delta) /(1-\alpha)$. Then we see that

$$
\begin{aligned}
E\left[\left|M_{n}\right|^{p}\right] \leq C_{p}\left(\sum_{i=1}^{n} \frac{\sigma^{2}}{i^{2 \alpha}}\right)^{(1+\delta))[2(1-\alpha)]} & \\
& +C_{p} \sum_{i=1}^{n} \frac{E\left[\left|Y_{i}-m\right|^{(1+\delta) /(1-\alpha)}\right]}{i^{[\alpha(1+\delta)] /(1-\alpha)}} .
\end{aligned}
$$

Since $\alpha /(1-\alpha)>1$, both terms above involve convergent series as $n \rightarrow \infty$. Thus, (3) holds.

4. $\alpha=1$ :

Using $M_{n}$ as defined in the previous case, we have that for some $\delta>0$,

$$
\begin{aligned}
E[N(t)]=\sum_{n=1}^{\infty} P\left(\left|M_{n}\right|>\log (n)\right) \\
\quad=\sum_{n=1}^{\infty} P\left(e^{\left|M_{n}\right|(1+\delta)}>n^{1+\delta}\right) \leq \sum_{n=1}^{\infty} \frac{1}{n^{\delta+1}} E\left[e^{\left|M_{n}\right|(1+\delta)}\right] .
\end{aligned}
$$

This last expression is finite whenever $\prod_{i=1}^{n}$ $E\left[e^{(1+\delta)\left|Y_{i}-m\right|}\right]$ is bounded.

5. $\alpha>1$ :

$$
E\left[N^{A}(t)\right]=\sum_{n=1}^{\infty} P\left(S_{n}<t\right)>\sum_{n=1}^{\infty}\left(1-\frac{E\left[S_{n}\right]}{t}\right)
$$


by Markov's inequality. Since $E\left[S_{n}\right]=m \sum_{i=1}^{n}$ $1 / i^{\alpha}$, we can ensure that the series in (4) diverges if we take $t>m \sum_{i=1}^{\infty} 1 / i^{\alpha}$.

REMARK 3: The moment condition in part 4 holds, for example, when $Y_{i}$ is exponentially distributed.

\section{QUALITATIVE BEHAVIOR OF THE PROCESSES-EXPONENTIAL CASE}

THEOREM 3: If $a<1$ and $Y_{1}$ is exponentially distributed with mean $m$, then

$$
\frac{a}{(1-a)} \log \left(\frac{(1-a) t}{m a}+1\right) \leq E\left[N^{G}(t)\right] \leq \frac{\log \left(\frac{(1-a) t}{m+1}\right)}{1-a} .
$$

PROOF: For $j=0,1,2, \ldots$, define $p_{j}(t)=P\left(N^{G}(t)=\right.$ $j)$. Then, a standard argument leads to

$$
p_{0}^{\prime}(t)=-\frac{1}{m} p_{0}(t)
$$

and

$$
p_{j}^{\prime}(t)=-\frac{a^{j}}{m} p_{j}(t)+\frac{a^{j-1}}{m} p_{j-1}(t) \quad(j \geq 1),
$$

from which it easily follows that

$$
\begin{gathered}
\frac{d}{d t} E\left[N^{G}(t)\right]=\frac{1}{m} E\left[a^{N G(t)}\right], \\
\frac{d}{d t} E\left[a^{N^{G(t)}}\right]=-\frac{(1-a)}{m} E\left[a^{2 N^{G}(t)}\right],
\end{gathered}
$$

and

$$
\frac{d}{d t} E\left[a^{-N^{G}(t)}\right]=\frac{(1-a)}{m a} .
$$

Since $E\left[a^{2 N^{G}(t)}\right] \geq E^{2}\left[a^{N^{G}(t)}\right]$, (7) gives

$$
\frac{d}{d t} E\left[a^{N G(t)}\right] \leq-\frac{(1-a)}{m} E^{2}\left[a^{N^{G}(t)}\right] .
$$

Solving this differential inequality, together with the initial condition $E\left[a^{N^{G}(0)}\right]=1$, we have

$$
E\left[a^{N^{G}(t)}\right] \leq \frac{1}{\frac{(1-a) t}{m}+1}
$$

which, together with (6), gives

$$
\frac{d}{d t} E\left[N^{G}(t)\right] \leq \frac{1}{(1-a) t+m} .
$$

Again, this can be solved, with $E\left[N^{G}(0)\right]=0$, to give the right-hand inequality of (5).

Similar arguing gives the left-hand side of (5):

$$
E\left[a^{N^{G}(t)}\right] \geq \frac{1}{E\left[a^{-N^{G(t)}}\right]}
$$

which, together with (6) and (8), gives

$$
\frac{d}{d t} E\left[N^{G}(t)\right] \geq \frac{1}{\frac{(1-a) t}{a}+m} .
$$

This, together with $E\left[N^{G}(0)\right]=0$ gives the result.

We can also obtain upper and lower bounds for $E\left[N^{A}(t)\right]$. Define $p_{j}(t)=P\left(N^{A}(t)=j\right)$ for $j=0,1,2, \ldots$ The usual arguments for continuous-time Markov chains lead to

$$
p_{j}^{\prime}(t)=-\frac{(j+1)^{\alpha}}{m} p_{j}(t)+\frac{j^{\alpha}}{m} p_{j-1}(t), \quad j=0,1,2, \ldots
$$

Multiplying through by $j$, and summing gives

$$
\frac{d}{d t} E\left[N^{A}(t)\right]=\frac{1}{m} E\left[\left(N^{A}(t)+1\right)^{\alpha}\right] .
$$

For $0 \leq \alpha \leq 1$, we have

$$
\frac{d}{d t} E\left[N^{A}(t)\right] \leq \frac{1}{m}\left(E\left[N^{A}(t)\right]+1\right)^{\alpha} .
$$

Solving this differential inequality with $E\left[N^{A}(0)\right]=0$ gives

$$
E\left[N^{A}(t)\right] \leq \begin{cases}(m t(1-\alpha)+1)^{1 /(1-\alpha)}-1, & \text { if } \alpha<1, \\ e^{m t}-1, & \text { if } \alpha=1 .\end{cases}
$$

To obtain a lower bound, we multiply (9) through by $j^{\alpha}$ and sum to obtain 


$$
\begin{aligned}
\frac{d}{d t} E\left[N^{A}(t)^{\alpha}\right]=\frac{\alpha(\alpha+1)}{2 m} E\left[\left(N^{A}(t)+1\right)^{\alpha} N^{A}(t)^{\alpha-1}\right] \\
\geq \frac{\alpha(\alpha+1)}{2 m} E\left[N^{A}(t)^{2 \alpha-1}\right] .
\end{aligned}
$$

If $\alpha \leq 1 / 2$, then we have

$$
\frac{d}{d t} E\left[N^{A \alpha}(t)\right] \geq \frac{\alpha(\alpha+1)}{2 m}\left(E\left[N^{A}(t)^{\alpha}\right]\right)^{2-1 / \alpha} .
$$

This can be solved together with the condition $E\left[N^{A \alpha}(0)\right]$ $=0$ to give

$$
E\left[N^{A}(t)^{\alpha}\right] \geq\left(\frac{t\left(1-\alpha^{2}\right)}{2 m}\right)^{\alpha /(1-\alpha)} .
$$

From (10), we have

$$
\frac{d}{d t} E\left[N^{A}(t)\right] \geq \frac{1}{m} E\left[N^{A}(t)^{\alpha}\right]
$$

which, with $E\left[N^{A}(0)\right]=0$, gives

$$
E\left[N^{A}(t)\right] \geq \frac{1-\alpha}{m}\left(\frac{1-\alpha^{2}}{2 m}\right)^{\alpha /(1-\alpha)} t^{1 /(1-\alpha)} .
$$

If $1 / 2<\alpha \leq 1$, then similar arguing, together with Jensen's inequality, leads to

$$
\frac{d}{d t} E\left[N^{A}(t)^{\alpha}\right] \geq \frac{\alpha(\alpha+1)}{2 m}\left(E\left[N^{A}(t)\right]\right)^{2 \alpha-1},
$$

which, together with (10), gives

$$
\frac{d^{2}}{d t^{2}} E\left[N^{A}(t)\right] \geq \frac{\alpha(\alpha+1)}{2 m^{2}}\left(E\left[N^{A}(t)\right]\right)^{2 \alpha-1} .
$$

The solution to this is

$$
E\left[N^{A}(t)\right] \geq\left[\frac{1-\alpha}{m} \sqrt{\frac{1+\alpha}{2}}\right]^{1 /(1-\alpha)} t^{1 /(1-\alpha)} .
$$

\section{PARAMETER ESTIMATION}

We next consider the problem of estimating model parameters as did Lam [7] for the geometric process model. Lam noted that

$$
\log \left(X_{i}\right)=\log \left(Y_{i}\right)-(i-1) \log (a)
$$

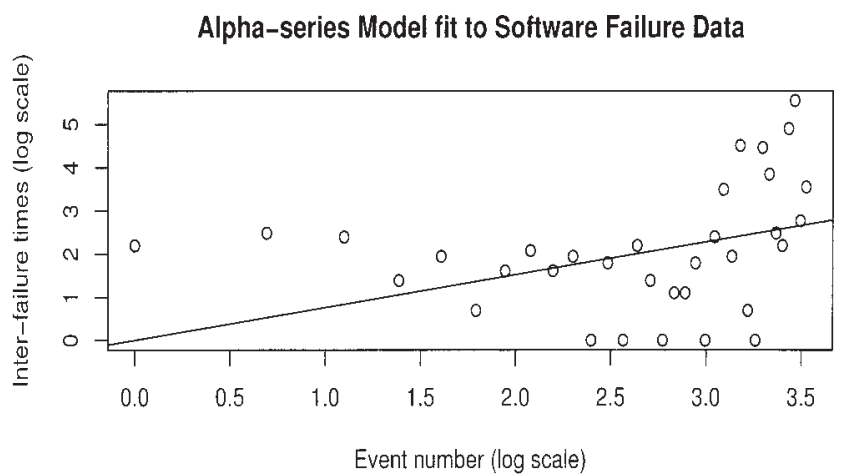

Geometric Model fit to Software Failure Data

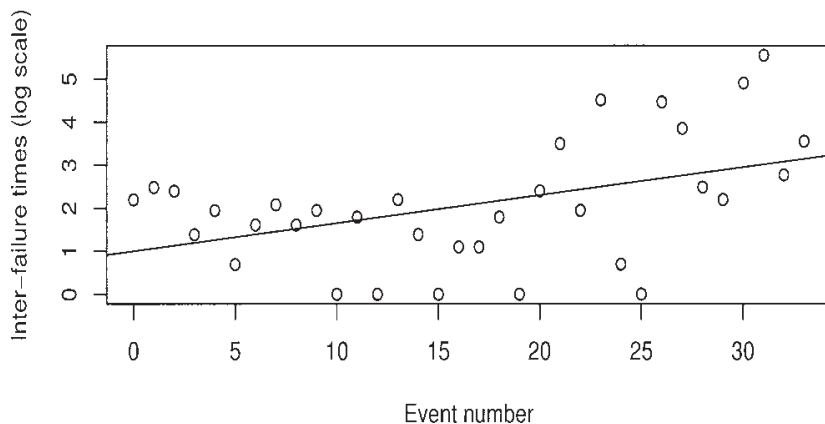

Figure 1. Least-squares fits of geometric and $\alpha$-series models to software failure data.

so that the least-squares estimate of the line relating $\log \left(X_{i}\right)$ to $i$ would lead to estimates of $\log (a)$ and hence $a$.

The estimation procedure for the $\alpha$-series process is very similar to that taken for the geometric process. The basic idea is as follows. If observed data is $X_{1}, X_{2}, \ldots, X_{n}$, then

$$
\log X_{n}=\log X_{0}-\alpha \log n=\beta-\alpha \log n+\varepsilon,
$$

where $X_{0}$ is a random variable with distribution $F(x)$, and $E\left[\log X_{0}\right]=\beta$. Linear least-squares can again be used to estimate $\beta$ and $\alpha$.

For an example of data where a stochastically increasing process might be appropriate, we consider the software failure data given in Crowder et al. [2]. In fitting the geometric process, we find that the estimated slope and intercept are .0650 (standard error $=.02, p$-value $=.005)$ and 1.00 , respectively. The data and fitted line are exhibited in the top half of Figure 1.

An estimate of $a$ can be obtained by exponentiating -.065 which gives us $\hat{a}=.937$. Thus, we are modeling the data with a stochastically increasing geometric process.

If the $\alpha$-series model is fit, the intercept is found to be not significant $(p$-value $=.22)$. The slope is .761 (s.e. .08, $p$-value $\left.=8 \times 10^{-10}\right)$ after dropping the intercept from the 

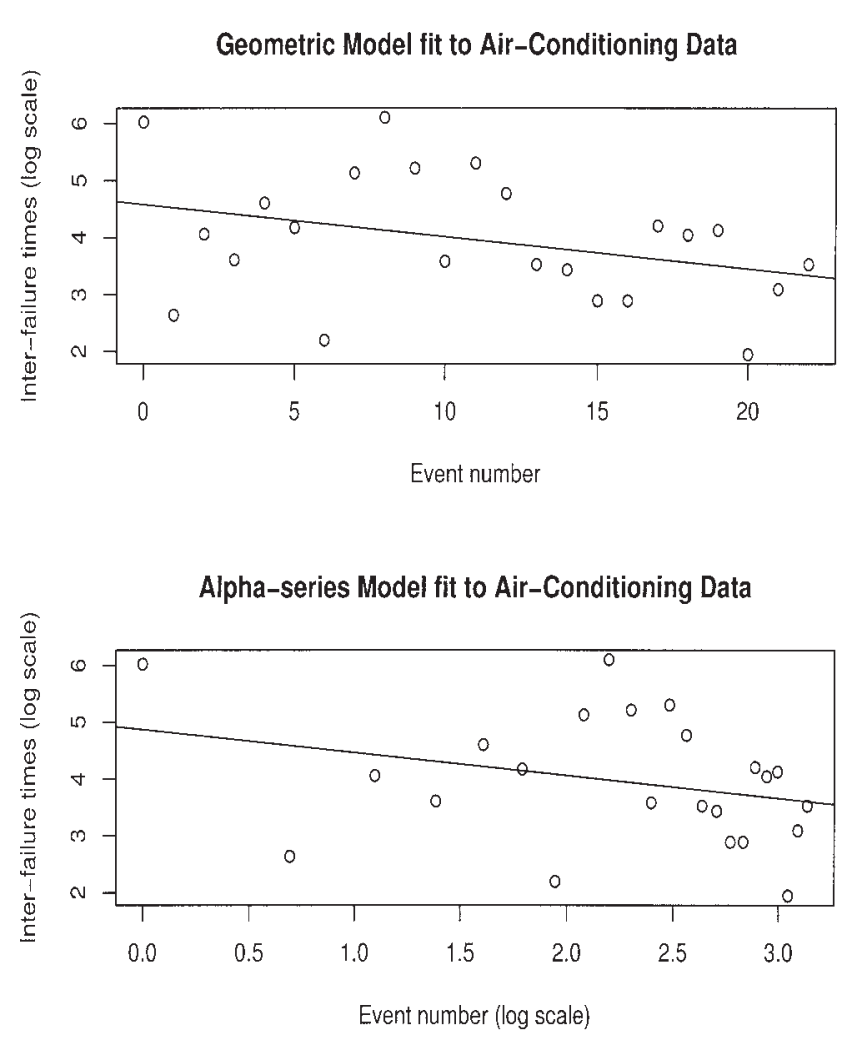

Figure 2. Least-squares fits of geometric and $\alpha$-series models to air-conditioning failure data.

model. The data and fitted line are exhibited in the bottom half of Figure 1. The estimate of $\alpha$ is $\hat{\alpha}=-.761$ which corresponds to a stochastically increasing $\alpha$-series process.

Figure 2 relates to an example where a stochastically decreasing process might be appropriate: the air conditioning failure data given in Crowder et al. [2]. When fitting the geometric model, we obtain slope and intercept estimates of -.0566 and 4.58 , respectively, leading to the estimate $\hat{a}=$ 1.06. This corresponds to the decreasing geometric process. We note that the standard error of the slope estimate is .033, corresponding to a $p$-value of .11. Thus, there is only one weak evidence that the process is truly decreasing. However, as pointed out in Section 3, if the process is actually decreasing, the estimated expected number of failures in any time interval is infinite, which does not seem reasonable.

Fitting the $\alpha$-series process, we obtain slope and intercept estimates of -.0404 and 4.87, respectively, leading to $\hat{\alpha}=$ .0404. Again, this corresponds to a stochastically decreasing process. This time, though, the expected number of failures in any finite time interval is finite as long as it is believable that the $2+\delta$ moment of the interfailure time random variable exists, for some $\delta>0$. Again, the $p$-value for the slope is too large (standard error $=.28, p$-value $=.16$ ) to state conclusively that the process is truly a decreasing process.

\section{NUMERICAL CALCULATION OF $E\left[N^{G}(t)\right]$ AND $E\left[N^{A}(t)\right]$}

When $a \leq 1$, there are few cases in which $E\left[N^{G}(t)\right]$ can be computed analytically. Therefore, an approximation technique is needed. For the renewal case $(a=1)$, this problem has been well-studied, and a stable solution method is given by Xie [12]. Chaudhry [1] has also given a useful method for solving (1) when $a=1$.

These methods will not work directly when $a<1$. However, an adaptation of the method of collocation (e.g., de Boor [3]), which is used for solving differential equations is one possible method of solution.

In contrast to the geometric process where a renewal equation analogue is available, it does not seem to be possible to obtain an integral equation whose solution is exactly $E\left[N^{A}(t)\right]$. The following inequalities can be obtained by conditioning on the value of $Y_{1}$.

THEOREM 4: If $E\left[N^{A}(t)\right]$ exists, then

$$
E\left[N^{A}(t)\right] \geq F(t)+\int_{0}^{t} E\left[N^{A}(t-x)\right] d F(x) d x
$$

and

$$
E\left[N^{A}(t)\right] \leq F(t)+\int_{0}^{t} E\left[N^{A}\left(2^{\alpha}(t-x)\right)\right] d F(x) d x .
$$

The solutions of these inequalities provide upper and lower bounds on $E\left[N^{A}(t)\right]$. Again, a collocation method may be the most appropriate approach.

Alternatively, to obtain an approximation to $E\left[N^{A}(t)\right]$, one could appeal to the following idea. Define a new family of processes, indexed by $k$, using

$$
S_{k, n}^{A}= \begin{cases}\sum_{i=k}^{n} \frac{Y_{i}}{i^{\alpha}}=\frac{Y_{k}}{k^{\alpha}}+S_{k+1, n}^{A}, & n \geq k, \\ 0, & n<k,\end{cases}
$$

and

$$
N_{k}^{A}(t)=\sup \left\{n: S_{k, n}^{A} \leq t\right\}
$$

Conditioning on $Y_{k}$ leads to: 
THEOREM 5: If $E\left[N_{k}^{A}(t)\right]$ exists, then for $k=1,2, \ldots$,

$E\left[N_{k}^{A}(t)\right]=\int_{0}^{t k^{\alpha}} E\left[N_{k+1}^{A}\left(t-\frac{y}{k^{\alpha}}\right)\right] d F(y)+F\left(t k^{\alpha}\right)$.

If the integrand above is replaced by $E\left[N_{k}^{A}\left(t-y / k^{\alpha}\right)\right]$, then an approximation to $E\left[N_{k}^{A}(t)\right]$ can be obtained. The quality of this approximation depends upon the condition

$$
\limsup _{k \rightarrow \infty}\left|E\left[N_{s \leq t}^{A}(s)\right]-E\left[N_{k+1}^{A}(s)\right]\right|=0 .
$$

When this condition holds, the following algorithm for approximating $E\left[N^{A}(t)\right]$ is certain to converge. (The approximation to $E\left[N^{A}(t)\right]$ is denoted by $\hat{E}\left[N^{A}(t)\right]$.)

1. Solve

$$
E\left[N_{k}^{A}(t)\right]=\int_{0}^{t k^{\alpha}} \hat{E}\left[N_{k}^{A}\left(t-\frac{y}{k^{\alpha}}\right)\right] d F(y)+F\left(t k^{\alpha}\right)
$$

for $\hat{E}\left[N_{k}^{A}(s)\right]$, for all $s \in\left[0, t k^{\alpha}\right]$.

2. For $i=k, k-1, \ldots, 2$, set

$$
\hat{E}\left[N_{i-1}^{A}(t)\right]=\int_{0}^{t^{\alpha}} \hat{E}\left[N_{i}^{A}\left(t-\frac{y}{i^{\alpha}}\right)\right] d F(y)+F\left(t i^{\alpha}\right) .
$$

The estimate for $E\left[N^{A}(t)\right]$ is given by $\hat{E}\left[N_{1}^{A}(t)\right]$. A simple bounding argument shows that

$$
\begin{aligned}
\sup _{s \leq t}\left|\hat{E}\left[N_{1}^{A}(t)\right]-E\left[N^{A}(t)\right]\right| \leq \sup _{s \leq t} \mid E\left[N_{k}^{A}(s)\right] \\
-E\left[N_{k+1}^{A}(s) \mid\right] F\left(k^{\alpha} t\right) F\left((k-1)^{\alpha} t\right) \cdots F(t) .
\end{aligned}
$$

This converges to 0 as $k \rightarrow \infty$ under condition (12).

\section{RELIABILITY AND SCHEDULING APPLICATIONS}

This section contains a brief discussion of some reliability and scheduling applications. An optimal $T$ policy and a flow-time minimization problem are described in which the decreasing geometric and $\alpha$-series processes are used to model machine uptimes.

First, we consider the problem of operating a deteriorating machine which is to be replaced when its total operating time reaches a prespecified level. This is the kind of problem (policy T) addressed by Lam [5]. We will show that the geometric model may not be appropriate, but the $\alpha$-series model can be used here.
Let $X_{i}$ denote the amount of time that the machine operates before its $i$ th failure, and let $N^{G}(t)$ denote the corresponding geometric process; since the machine is deteriorating, we assume that the process is decreasing with parameter $a=1 / b$. The expected working age of the machine at time $T$ is then given by

$$
E\left[\sum_{i=1}^{N^{G}(T)} X_{i}\right]=m\left(\frac{1-E\left[b^{N^{G}(T)}\right]}{1-b}\right) \leq \frac{m}{1-b} .
$$

If the repair times $R_{i}$ have mean $m_{R}>0$ and are independent and identically distributed and independent of $N^{G}(t)$, then it follows from Theorem 1 and the subsequent remark that the expected length of a replacement cycle is infinite. The expected cost of a cycle is given by

$$
E\left[-T+\gamma \sum_{i=1}^{N^{G}(T)} R_{i}\right]+\gamma_{R}=\gamma_{R}-T+\gamma E\left[\sum_{i=1}^{N^{G}(T)} R_{i}\right],
$$

where $\gamma$ is the repair cost rate, and $\gamma_{R}$ is the replacement cost. By Theorem 3, this quantity must also be infinite. Thus, the decreasing geometric model is not suitable if we desire an optimal $\mathrm{T}$ policy. This has already been pointed out by Stadje and Zuckermann [10], but for different reasons.

We next consider this optimal T policy using the $\alpha$-series model. We assume that the repair periods are i.i.d., and we want to choose $T$ to minimize

$$
\frac{E[\text { cost }]}{E[\text { cycle length }]}=\frac{-T+\gamma E\left[N^{A}(T)\right] E\left[R_{i}\right]+\gamma_{R}}{T+E\left[N^{A}(T)\right] E\left[R_{i}\right]},
$$

where $R_{i}$ is the length of the $i$ th repair period. Under the conditions of Theorem 3.2, this has a nontrivial solution. This solution can be obtained numerically, using the methods of Section 5.

The second problem we wish to discuss concerns the scheduling of a set of $M$ independent jobs (with mean processing times $m_{i}$ ) on a deteriorating machine so as to minimize the expected flow time (see Ross [9]). If the machine uptimes $Y_{i}$ follow a decreasing geometric process, and downtimes are independent and identically distributed with mean $m_{D}$, then the expected flow time is

$$
\sum_{i=1}^{M} E\left[C_{i}\right]=\sum_{j=1}^{M}(M-j+1) m_{j}+\sum_{i=1}^{M} m_{D} E\left[N^{A}\left(\sum_{j=1}^{i} Y_{j}\right)\right]
$$

where $C_{i}$ is the completion time of job $i$. By Theorem 1 , the expected flow time is infinite. Thus, the geometric model is, again, not suitable. 
We next consider this scheduling problem using the $\alpha$-series model: Minimize the expected flow time in case of $M$ independent jobs, $Y_{1}, Y_{2}, \ldots, Y_{M}$ with means $m_{i}$. Machine uptimes follow the decreasing $\alpha$-series process, and downtimes are i.i.d. with mean $m_{D}$.

The expected flow time is

$$
\sum_{i=1}^{M} E\left[C_{i}\right]=\sum_{j=1}^{M}(M-j+1) m_{j}+\sum_{i=1}^{M} m_{D} E\left[N^{A}\left(\sum_{j=1}^{i} Y_{j}\right)\right]
$$

and

$$
N^{A}\left(\sum_{j=1}^{i} Y_{j}\right) \doteq\left((1-\alpha) \frac{\left(\sum_{j=1}^{i} Y_{j}\right)}{m}\right)^{1 /(1-\alpha)}
$$

Therefore, minimizing expected flowtime involves the $1 /(1-\alpha)$ moments of the job processing times. In cases where $E\left[Y^{1 /(1-\alpha)}\right]$ increases with $m_{j}$, the expected flow time is minimized when $m_{1}<m_{2}<\cdots<m_{M}$.

\section{CONCLUDING REMARKS}

The geometric process, $N^{G}(t)$, is an interesting and potentially useful model for deteriorating machines. It is tractable and easy to use. In this paper, we have introduced an alternative model, the $\alpha$-series process $N^{A}(t)$, which shares these characteristics. The two processes may be viewed as complementary to one another. For example, the increasing geometric process may be appropriate for modeling machine downtimes, while the $\alpha$-series process can be used for modeling machine uptimes.

Both the increasing geometric and the increasing $\alpha$-series processes have a finite first moment under fairly general conditions. However, the decreasing geometric process usually has an infinite first moment. The decreasing $\alpha$-series process has a finite first moment under fairly general conditions, if $\alpha \leq 1$. Thus, for modeling successive uptimes, the decreasing $\alpha$-series process will have wider applicability than the decreasing geometric process.

To gain some appreciation for the qualitative behavior of both processes, we studied the exponential case in some detail and showed that the increasing geometric process exhibits logarithmic growth, while the decreasing $\alpha$-series process exhibits power law and exponential growth, depending upon whether $\alpha<1$ or $\alpha=1$. This is a further indication as to how the $\alpha$-series process provides a complementarity aspect: In the exponential case, the geometric process only allows for logarithmic growth or explosive growth, but nothing in between.

Statistical inference can be done for both processes equally easily, using a least-squares approach. We have demonstrated how both the increasing and decreasing models (for both types of processes) fit actual data.

As a further demonstration of the tractability of the processes, we have suggested algorithms for computing numerical approximations for $E\left[N^{A}(t)\right]$ and $E\left[N^{G}(t)\right]$ where these moments exist. Specifically, it is possible to obtain numerical approximations as solutions of integral equations or systems of integral equations. In both cases, the computations are not prohibitive. These approximations find application in formulas arising in reliability and scheduling applications.

As for the case of renewal processes, asymptotic approximations to $N^{A}(t)$ and $E\left[N^{A}(t)\right]$ can also be obtained. These will be the subject of another paper.

We have demonstrated the applicability of the decreasing $\alpha$-series process in situations where the geometric process may not be as useful because of the nonexistence of $E\left[N^{G}(t)\right]$. We have attempted to give some scope to the variety of applications in reliability and scheduling for this alternative model, but there are many potential applications that we have not addressed in this paper.

\section{ACKNOWLEDGMENTS}

This work was supported by research grants from the Natural Sciences and Engineering Research Council of Canada (NSERC). Wei Li would also like to acknowledge that his work was partially supported by the National Natural Science Foundation of China (NNSFC) with Contract No. 60474067. The authors are grateful to Hao Yu for assistance with the proof of Theorem 3.2, and they acknowledge the very useful comments of an associate editor as well as two referees, which led to a substantial improvement in the paper.

\section{REFERENCES}

[1] M.L. Chaudhry, On computations of the mean and variance of the number of renewals: A unified approach, J Oper Res Soc 46 (1995), 1342-1364.

[2] M.J. Crowder, A.C. Kimber, R.L. Smith, and T.J. Sweeting, Statistical analysis of reliability data, Chapman and Hall, London, 1991.

[3] C. de Boor, A practical guide to splines, Springer, Berlin, 1978.

[4] W. Feller, An introduction to probability theory and its applications, Wiley, New York, 1971, Vol. II. 
[5] Y. Lam, A note on the optimal replacement problem, Adv Appl Probab 20 (1988), 479-482.

[6] Y. Lam, Geometric processes and replacement problem, Acta Math Appl Sinica 4 (1988), 366-377.

[7] Y. Lam, Nonparametric inference for geometric processes, Comm Statist Theory Methods 21 (1992), 2083-2105.

[8] Y. Lam and Y.L. Zhang, Analysis of a two-component series system with a geometric process model, Naval Res Logist 43 (1996), 491-502.
[9] S.M. Ross, Stochastic processes, Wiley, New York, 1983.

[10] W. Stadje and D. Zuckerman, Optimal strategies for some repair replacement models, Adv Appl Probab 22 (1990), 641-656.

[11] W.F. Stout, Almost sure convergence, Academic Press, New York, 1974

[12] M. Xie, On the solution of renewal-type integral equations, Commun Statist Simulation 18 (1989), 281-293. 